\title{
Planarity Testing of Graphs on Base of a Spring Model
}

\author{
Günter Hotz and Steffen Lohse \\ Universität des Saarlandes - Fachbereich Informatik, Saarbrücken, Germany \\ http://www-hotz.cs.uni-sb.de
}

\section{Short Description}

It is well known that planar embeddings of 3-connected graphs are uniquely determined up to isomorphy of the induced complex of nodes, edges and faces of the plane or the 2-sphere 11. Moreover, each of the isomorphy classes of these embeddings contains a representative that has a convex polygon as outer border and has all edges embedded as straight lines. We fixate the outer polygon of such embeddings and regard each remaining edge $e$ as a spring, its resilience being $|e|^{k}(|e|$ euclidean length of $e, k \in \mathbb{R}, 1<k<\infty)$. For 3-connected graphs, exactly one power-balanced embedding for each $k$ exists, and this embedding is planar if and only if the graph with the fixated border polygon has a planar embedding inside that very polygon. For $k=1$ or $k=\infty$, some faces may be collapsed; we call such embeddings quasi-planar 2. It is possible to decide the planarity of any graph embedding in linear time 3. The motivation for this result was to develop a planarity test that simultaneously with the decision process constructs a concrete planar embedding. This algorithm should work in three steps:

1. Choose any circle of the graph and embed it as a convex polygon in the plane or as an equator of the 2 -sphere.

2. Fixate this circle and hang in the rest of the graph. Substitute each edge by a spring of a fixed norm $k$ and compute its power-balanced position.

3. Decide if the computed embedding can be decomposed into two planar flanks. The graph is planar if and only if this is possible. On the sphere, these flanks should be represented by the two hemispheres belonging to the fixated circle.

If the input graph originates from a hierarchical definition of a chip layout system (e.g. 4]), that definition gives the base for an efficient algorithm to compute this embedding by use of a multi grid method [5].

The algorithm presented here assumes the graph to be 3-connected - but it does not make special assumptions on its definition. We only use the isomorphy theorem mentioned. The stepwise construction of the embedding reflects the single step method of iterative solutions of equations. Some steps produce quasiplanar embeddings which in later steps will be unfolded to planar embeddings by hanging in more springs. This requires some complex data structures, the handling of which is responsible for the high worst case runtime bound $O\left(n^{2} \log n\right)$ of the algorithm. The proof of this bound assumes a worst case situation which 
seems never to appear. We assume that a more thorough analysis will come out with a $O(n \log n)$ bound.

We ran tests on randomly generated planar graphs with up to 6000 nodes and compared the running time with the algorithms Fáry [6] $\left(O\left(n^{2}\right)\right)$ and Schnyder [7] $(O(n))$ implemented in [8]. In all examples, our algorithm was in the worst case and the average case faster than Fáry, and Schnyder always was faster than our algorithm. Our algorithm shows complexity jumps for graphs of size $n$ at $2^{n}$, suggesting a possible additional speedup. The pictures produced by our algorithm, representing the planar embeddings on the sphere, have a certain aesthetical attraction.

The detailed description of the algorithm can be found in the Master's Thesis of the second of the authors [9]. It includes a $\mathrm{C}++$ implementation for MS Windows and UNIX.

\section{Screenshots}
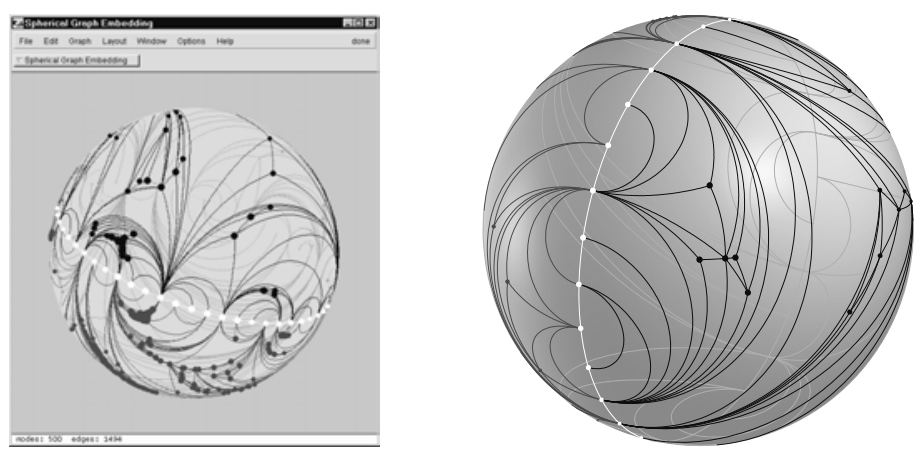

\section{References}

1. Hotz, G.: Einbettung von Streckenkomplexen in die Ebene. Math. Annalen 167, 214-223 (1966)

2. Becker, B., Hotz, G.: On the Optimal Layout of Planar Graphs with Fixed Boundary. SIAM Journal on Computing 16(5), 946-972 (1987)

3. Becker-Groh, U., Hotz, G.: Ein Planaritätstest für planar-konvexe Grapheneinbettungen mit lin. Komplexität. Beiträge zur Algebra u. Geom. 18, 191-200 (1984)

4. Hotz, G., Reichert, A.: Hierarchischer Entwurf komplexer Systeme. Chapter 8 in: Wegener, I. (Ed.): Highlights aus der Informatik. Springer-Verlag (1996)

5. Osthof, H.-G.: Optimale Grapheinbettungen und ihre Anwendungen. Dissertation. Universität des Saarlandes (1990)

6. Fáry, I.: On Straight Line Representing of Planar Graphs. Acta Sci. Math. 11, 229-233 (1948)

7. Schnyder, W.: Embedding Planar Graphs on the Grid. Proc. 1st ACM-SIAM Symp. on Discrete Alg., 138-148 (1990)

8. Mehlhorn, K., Näher, S., Seel, M., Uhrig, C.: The LEDA User Manual (Version 3.7). Max-Planck-Institut für Informatik, Saarbrücken (1999)

9. Lohse, S.: Ein Planaritätstest und Einbettungsverfahren für Graphen. Diplomarbeit. Fachbereich Informatik, Universität des Saarlandes (2001) 